\title{
Mindfulness and Complex Identities in Equity Training: A Pilot Study
}

\author{
Mónica J. SÁNCHEZ-FLORES \\ Department of Sociology and Anthropology, Faculty of Arts, Thompson Rivers University (TRU) \\ (C) 2017 Mónica J. Sánchez-Flores \\ This is an open access article distributed under the Creative Commons Attribution-NonCommercial-NoDerivs license \\ (http://creativecommons.org/licenses/by-nc-nd/3.0/) \\ DOI: 10.1515/eras-2017-0002
}

\begin{abstract}
Equity (diversity, anti-oppression) training refers to teaching self-awareness of social location, privilege/disadvantage that mainstream culture perpetuates, and working self-reflectively for equity in everyday interactions amid diversity. This paper investigates the efficacy of equity training on the basis of mindfulness, compassion, and an intersectional/complex identity. Mindfulness refers to a vivid awareness of the present moment, of one's own embodiment, thoughts, and emotions, fostering self-awareness and compassion. A complex conception of identity in an intersectional approach recognizes that people hold multiple statuses and I propose that this helps them self-identify with both privilege and disadvantage beyond dichotomic notions, finger pointing, and angry reactions. I first discuss the relevant theory of intersectionality and compassion, and consider the significance of this kind of approach to equity training. I then report the outcome of my pilot study that compares the impressions of two small groups of trainees in different equity workshops who were interviewed: The target group attended a workshop based on mindfulness exercises within an intersectional framework, and the comparison group attended a workshop not based on mindfulness, but that included information about privilege, disadvantage, and compassion. The findings point to an enhanced and emotionally involved self-awareness in respondents that participated in the target group workshop, based on mindfulness and empathy enhancing exercises.
\end{abstract}

Keywords: equity training, diversity, inclusiveness, compassion, mindfulness, intersectionality

\section{Introduction}

Equity training refers to teaching about awareness of the different sources of privilege and disadvantage that mainstream culture perpetuates; people's own social location with respect to privilege and disadvantage, and how to engage in a constant self-reflective effort to work towards equity in everyday interactions amid diversity. As a facilitator of equity workshops, I have found that when participants clearly inhabit dimensions of privilege (as in heterosexual, white, male persons, for example), they often resist being seen as sources of oppression. This produces dissonance when this kind of participant also inhabits invisible dimensions of disadvantage (having grown up poor or being homosexual, for example) while at the same time being labeled "privileged." This dissonance appears also when a person in a minority group resists being portrayed as disadvantaged (for example, a racialized person with class privilege). In these cases, I have found that framing this training in the awareness that one can inhabit both privilege and disadvantage at the same time can soften resistance to being reflective on the consequences of social location. This speaks to the complexity of human identity and how we can inhabit realms of privilege and disadvantage at the same time; which I frame in an expanded notion of intersectionality that I discuss below. Another realm of resistance to self-reflection and self-awareness comes from the strong emotions that being 
identified as privileged (or disadvantaged) may elicit. As my career as an equity educator started, I thought it would be enough to engage workshop participants in a discussion of the importance of compassion to equity, to help them see its relevance. I thus designed my equity training workshops based on the discussions of love and compassion, and gave participants some reflective exercises that would engage them in considering this moral orientation. This approach did elicit some positive feedback, yet the work remained conceptual and detached, participants kept identifying the issues of lack of fairness as a societal problem and not as something that they themselves were involved with or that emanated from their own prejudice. I eventually came in touch with some popular literature on mindfulness and mindful meditation, and in the media, listening to radio and video on the ways meditation has been used in certain educational settings. With this in mind, I eventually integrated mindfulness exercises in my equity training workshops, whose effectiveness I explore in this pilot study.

The topic of how to effectively teach for equity and celebration of diversity has become an important part of my research and my teaching practice. In the spirit of being selfreflective, I will disclose that I am a middle class, well educated, woman of color from Mexico, an immigrant in Canada, with a close relationship with racism; having been bullied for being too brown while attending a private school in Mexico City where most children were white (or what Mexicans perceive to be 'white'). This had a great influence in my formation; but the one aspect of growing up in Mexico that most affected my intellectual lifechoices was a keen awareness of belonging to an underdeveloped country-looked down on by the developed world. My academic life started with theoretical work that inquired into the possibility of ways of knowing that are alternative to the western/intellectual traditions of science and humanities. Upon reflection I think this had to do with my desire to look beyond the western masters for sources of being and knowing that did not consider my people underdeveloped. In my doctoral dissertation, I explored research on human cognition, especially its emotional aspect as essential for our formation as persons and social beings. Part of my thesis was published in my first book, Political Philosophy for the Global Age (2005) and I developed these ideas further in my Cosmopolitan Liberalism; Expanding the Boundaries of the Individual (2010). These books argue that the moral principle of compassion will be the guide for liberalism to shed its supremacist and colonial biases. In 2009 , I began to train people about equity, diversity, and inclusion as well as prejudice and discrimination. I was given the task and challenge to prepare teachers in my university's Master in Education program on how to approach the issue of equity in their increasingly diverse classrooms. In this process, I learned that this was only possible by making participants aware of their own social location and prejudice through self-reflective exercises. I put together an equity workshop for this course where the focus was to create an awareness that nobody is without prejudice (Horn, 2008, Brookfield, 2014). This is how I became an equity educator. My theory work on compassion is always a referent behind my reflections and teaching on the topic of equity.

\section{Theoretical Framework}

Sources of privilege and disadvantage come from what sociologists call "ascribed statuses," social categories given to us by society at birth (such as race, ethnicity, gender, ability, or social class), where collective conceptions strongly condition and mold our senses of who we are; they produce both group boundaries and social hierarchies (Anthias, 2012). As social beings, we think of these categories as inevitable, 'natural,' or emanating from our biology; yet they entail social constructions that have a subconscious effect on how we perceive and treat each other each other on a daily basis. In his book Deep Diversity, Choudhury (2015) 
speaks of "implicit bias," or mental associations of an ascribed status; the higher or lower standing of the status is so entrenched in our culture and our socialization/perception that they operate without our awareness intention or immediate control. These perceptions require that we make the conscious effort to "re-program" ourselves. Being perceived as female and/or as racialized (or both), for example, produces layers of disadvantage and creates an uneven "playing field" that equity training aims to bring to awareness. This awareness, however, is always mediated by a sense of fairness in liberal-capitalist societies, where people believe in meritocracy. Individual success is seen as the product of one's own effort and not necessarily of the privilege that people get when they belong in certain groups (such as being male or white, or both). Merit and individual achievement are often used by workshop participants as points of resistance, since equity training involves recognition of how ascribed statuses can be sources of undeserved privilege.

As an equity trainer and educator, I have found that in encountering this resistance, it helps to focus on the complexity of human identity-the multiplicity of sources that we resort to in order to put together our sense of who we are and also how we inhabit both privilege and disadvantage at the same time. I introduce this complexity in workshops by talking about my own privilege. The visible aspect of my identity is that I am a brown-skinned woman (with a Spanish accent) and I explain how the intersection of origin, race, and gender has been a source of disadvantage for me at times. However, an invisible aspect of my privileged identity (or at least, not immediately perceptible) is that I grew up in a middle class family, in a protective environment, where I received an excellent education. Modeling vulnerability and opening up a discussion on the sources of my own identity allows for participants to start thinking about the sources of their own identity and privilege/disadvantage (that are not always evident). Since some people resist being identified as oppressors or as holding undeserved privilege, it helps to frame this reflection by acknowledging that identities are complex, multifaceted, and multi-layered. This approach has helped me frame workshop discussions to present an idea of fairness that people can identify with when considering sources of privilege and disadvantage.

Intersectionality is a theoretical approach that emerged relatively recently in equity, social justice, and diversity studies. The origins of this approach are in black feminist thought, when African American women felt alienated by both black men's and white women's movements. Black feminists tried to conceptualize their own experience as different from these other two groups, each of which focus on a single dimension of inequality (Gopaldas, 2013). This led to a richer conceptualization of marginalized minorities occupying multiple dimensions of disadvantage within systems of oppression. Kimberle Crenshaw coined the term intersectionality as a metaphor to illustrate how multiple systems of oppression are sometimes like road intersections, "whereby the racism road crosses with the streets of colonialism and patriarchy, and 'crashes' occur in the intersections... there is a double, triple, multiple, and many-layered blanket of oppression" (Dhamoon, 2011, p. 231). This way of looking at ascribed statuses acknowledges their complexity as well as that of the societal context in which identity occurs. Patricia Hill Collins (2000) is credited with acknowledging that there are macro-level systems she calls "interlocking oppressions" that link multiple sources of subjugation. For example, being gendered, racialized, and poor at the same time; this simultaneity produces a different experience than any form of discrimination that is seen as standing on its own. In highlighting the multiplicity of human identity and its many layers of inequality, intersectionality provides a way to correct previous approaches that tended to essentialize and homogenize singular identity constructs (Anthias, 2012). Three relevant aspects of this approach are that it focuses on the multiple positioning of people's identity, the relational nature of social positioning, as well as the power relations that define whether they 
entail privilege or disadvantage (Dhamoon, 2011). This means that the way people form their sense of self does not happen apart from other people immersed in structures of power. Such structures influence subject formation and also social identity structures are often interdependent (Gopaldas, 2013).

This has brought to the fore awareness that multiple sources of subjugated identities have multiplicative effects in terms of disadvantage; but it has also highlighted how some oppressive ascribed statuses that produce disadvantage may at times coexist with privilege. The co-existence of privilege with disadvantage and their visibility/invisibility are particularly relevant aspects of seeing identity as complex and multi-layered in equity training. This helps participants see how they themselves are never absolutely oppressive nor absolutely disadvantaged. When using this approach together with auto-ethnographic methods in the exploration of identity, it has been found that there is both a relationship, but also a tension between privileged and disadvantaged identities (Jones, 2009). When looking at the complexities of lived experience, it is important to see that people inhabit several societal dimensions and categories and they can all have an impact on identity formation and social interaction. If this complexity goes unseen, one-dimensional perspectives may rob equity trainers from the chance to engage participants and garner their commitment with equity and fairness, especially when talking about race.

Racial categories are problematic to many participants in anti-racist education, because individuals do not necessarily fit neatly into racial categories. Gnanadass (2014) tells us: "One of my students was half Peruvian and half Egyptian. Another was African American, Indian, Chinese, and British. What were their races? How did they identify and how were they identified?" (p. 97). At the same time, when speaking about race, white people may feel uncomfortable and guilty due to their perceived privilege. What DiAngelo (2011) calls "white fragility," refers to the catering to such comfort and the rejection of "racial stress" exhibited by interactions with white participants in anti-racist training. Such training with participants perceived as white may trigger "a range of defensive moves [that] include outward display of emotions such as anger, fear, and guilt, and behaviors such as argumentation, silence, and leaving the stress-inducing situation" (DiAngelo, 2011, p. 57). Reports of defensive behavior in equity (or anti-oppression, or anti-racism) training are legion. I think that these types of emotional reactions to equity or diversity training are prompted by approaching this training on the basis of single issues - such as race. When participants in equity training are asked to consider as many dimensions of identity that can be sources of privilege and disadvantage as possible, they get the opportunity to negotiate their understanding of their own identity as oppressive to others; but also, at the same time, they get the chance to acknowledge realms of disadvantage for themselves. "The relationship between privileged and oppressed identities," Jones tells us, "illuminates the significance of the intersections, intersections that both construct identities and complicate them" (2009, p. 296).

I would like to propose that such intersections are of the essence in equity training. The complex ways in which identity formation and negotiation takes place, mirrors the complex ways in which privilege and disadvantage can converge in the same person. It is very hard to see and acknowledge one's own privilege. People with privileged identities often write about the epiphany of realizing that they are oppressive to others and in what way. Consider the intimations of Peggy McIntosh in her now classic "White privilege: Unpacking the invisible knapsack," written in 1988, excerpts of which have been reprinted in journals and online sources, also for anti-oppression training purposes. In her paper, McIntosh (1990) details the many ways her white skin color has given her unearned privileges, how "an invisible weightless backpack of special provisions, maps, passports, codebooks, visas, clothes, tools and blank checks" (p. 31) gave her privileges in contrast to her African 
American sisters. In a video on this same subject, McIntosh tells us that to obtain the awareness of her white privilege, she had to ask her unconscious mind about her own bias, to "pray on it" (Spectrumlens, 2008). She explains how this awareness came to her in the middle of the night, had to get up and write the points of privilege down, or they would be gone by the next day. I use this as an example to illustrate how hard it is to gain an awareness of one's own privilege and "own" it, even in willing subjects. For this reason, I started using an abstract compassionate approach and eventually adopted mindfulness strategies and exercises in my equity training workshops: To have tools that would allow people to own their privilege and not reject the idea outright that they have this privilege (an awareness of which produces discomfort).

The compassionate approach is basically a moral orientation in contradistinction to a judgmental type of morality or principled morality that is more commonly resorted to when discussing equity. In his book Deep Diversity, Choudhury (2015) tells us about his own experience with this kind of morality, represented by his difficulty dealing with the power dynamics among anti-racism trainers and his activist social justice community. He tells us how he eventually experienced a personal burn-out and had to walk away from his finger pointing angry activism to embark in a self-healing journey that took him to embracing compassion and mindfulness in order to go back to his equity work again. The judgmental type of approach on its own conveys anger and resentment, blame and finger-pointing, and also self-righteousness when privilege is owned; while these may be legitimate feelings and behaviors, they may become counterproductive in equity work. I believe that unmanaged emotional stress is one of the main reasons why equity or anti-racism or anti-oppression training winds up being ineffectual. Guilt is an interesting emotion associated to a judgmental moral orientation (Sánchez-Flores, 2010); it has been seen as a two-pronged moral emotion: It alerts people of what norm or social value is being violated by their behavior, but people can also experience guilt when there is no apparent transgression (Carni et al., 2013). The former kind is seen as more pro-social and based on one's own behavior; but the latter kind can lead to psychopathologies or, as Carni et al. (2013) tell us, "people may feel guilty when they fare better than other people, even if they are not responsible for the inequity" (p. 334). I do not propose that this moral orientation should be eliminated from equity training, as I said above, guilt gives people clarity about their own prejudice on ascribed social locations (race, gender, sexuality, ability, etc.). However, on its own, a judgmental moral orientation prevents commitment with equity: It reinforces a sense of guilt in people who see themselves as occupying privileged positions in their social location. Such people may explain their privilege on the basis of merit (deeply embedded in our culture) to resist the discomfort. I want to stress that I do not advocate to simplistically "save" privileged people from the pain of becoming aware of their undeserved goodies, of "owning their privilege." To address this, I think that equity educators ought to be equipped with some strategies to deal with this guilt and discomfort as it emerges in the name of building more equitable communities. After all, such discomfort and guilt are necessary in the dismantling of privilege; but we need to have tools to deal with them so that they do not morph into anger that rejects equity (however wrong or misinformed).

I propose a phenomenological approach that embraces both principled (judgmental) morality as well as what I call compassionate morality (Sánchez-Flores, 2010). Compassion has been defined as an emotion that can guide our moral decisions that we experience when we are aware of somebody else's undeserved misfortune (Goetz et al., 2010, Nussbaum, 2001), and has also been seen as an emotion that can be fruitfully directed to oneself (Neff, 2003, Neff et al., 2007a, Neff et al., 2007b). In my Cosmopolitan Liberalism (2010), I have discussed the conception of compassionate morality, or a "hermeneutic of the heart," an 
understanding of our shared humanity and vulnerability that addresses the needs of universal cosmopolitanism: what human beings owe one another. This is based on the ideal of universal love that extends to all human beings and beyond (non-anthropocentric); to include the oppressor or any potential oppressors. My political theory proposes a revolution of the heart that can only take place at an individual and private level in self-reflection about our emotions. This is based on an awareness of the complexity of our identities that produces sophisticated cultures of care based on freedom from hate and fear within those cultures (Sánchez-Flores, 2010). In equity work, compassionate morality can be the basis to think about how we ought to address each other in daily interaction for the purposes of creating fair and equitable communities. Compassionate morality allows for people to reflect on the humanity of the people that they interact with on a day to day basis. This involves developing compassion for others and deploring that anyone should suffer greatly and undeservedly, but since everyone has the potential of being perceived as an oppressor, it also involves allowing feelings of compassion for oneself.

Self-compassion is fruitful when dealing with the kind of guilt that people experience due to their privileged social location ascribed to them by societal structures that they themselves did nothing to produce - but from which they benefit nonetheless. The literature confirms that equity work must recognize that there is an "uneven playing field" and this entails realizing that we can be part of the system that creates suffering as we reflect on our own privilege in an inequitable world. Hernández-Wolffe \& McDowell (2012) found that this was the case with family therapists who worked with people in marginalized positions, as "[i]increased awareness [of their privilege] involved personal struggles with guilt" (p. 175). Awareness of privilege requires challenging our own prejudice that is constantly confirmed by the dominant culture (for example, in media representations of desirable human characteristics) and is based on a wide-spread belief in individuality and meritocracy. Guilt can work as a signal that may trigger awareness of privilege, and this is why I have said before that principled or judgmental morality is an orientation that is needed in equity work. However, guilt is not sufficient on its own to elicit commitment to equity and can even turn people off equity work. This is where self-compassion is useful to deal with guilty feelings associated to our undeserved privilege. According to Neff et al.(2007b), compassion for oneself involves being kind and understanding to our human condition that includes suffering and our own perceived inadequacy. It also involves mindfulness about our own emotions or the ability to "face (rather than avoid) painful thoughts and feelings, but without exaggeration, drama or self-pity" (Neff et al., 2007b, p. 908-909).

Making equity trainees aware of their implicit bias may bring up feelings of guilt, but it is important to deal with them to "own" our privilege. Racist, sexist, able-ist, and many other forms of prejudice become part of us as we consume main-stream culture and media. We internalize such prejudice if we do not engage in constant self-reflection, it becomes unconscious, "implicit bias," about which people are unaware (Choudhury, 2015). This is why equity work is a "labor of love." It is a constant practice, an endeavor that is never done. We ought to be vigilant of how shared meanings and ideas about what is desirable or proper may produce boundaries and hierarchies, which exacerbate power relations to the benefit of some and detriment of others. Self-reflectivity may bring us awareness of our own undeserved privilege and our inadequacy in having internalized the prejudice of the dominant culture. In contrast to guilt, self-compassion allows participants to accept and forgive their own imperfection; be aware of shared humanity in that imperfection. This opens up the possibility of a less judgmental attitude towards one-self and a more objective approach to our own emotions.

The literature on compassion reveals a relationship to mindfulness, which can be seen 
as a practical strategy to further compassion. Mindfulness is a vivid awareness of the present moment, of one's own embodiment, thoughts, and emotions, fostering self-awareness. It is a practice that relies on specific methods of breathing, calm mental states, and focusing the mind in the present moment. It has been used as an actual tool that has been recently gaining ascendancy and support for good health and general wellbeing (Holas \& Jankowski, 2013, Neff \& Germer, 2013, Galante et al., 2014), and in different contexts, such as pro-social behavior in pre-school children (Flook et al., 2015), elementary school students' behavior(Schonert-Reichl et al., 2015), and also in dealing with various forms of depression, over-eating, parenting, cognition, other uses can be found in the relatively new interdisciplinary journal called Mindfulness, published by Springer. The following pilot study on equity training applied some tools that my research assistants and I designed to further self-awareness through mindfulness in equity workshops.

\section{Methodology}

The project was put together by a team of researchers including me as principal investigator (PI) and two research assistants. I was in charge of designing and delivering the workshops (a consultant helped us with the guided meditation during workshop delivery), and my research assistants helped with exercise design and layout and in organizing the data and coding it. The project was completed with 17 respondents who were recruited with an open invitation to employees (staff and faculty) to a one hour workshop on equity and compassion in my university of adscription. More than 17 people participated in the workshops (approximately 50 participants in two workshops), but only 17 people agreed to be interviewed for this research project. Two different equity workshops were offered: both workshops focused on equity and compassion, both provided information and definitions of the main concepts, but only one of them involved mindfulness exercises. Both groups of respondents were interviewed after participating in either workshop following semi-structured interviews, where they were asked what they took away from the workshop, how they conceived of equity, and examples of equity or inequity in the workplace; how important emotions were in equity work in general to them, and also whether compassion was particularly important when thinking about equity. Different participants completed each workshop and were interviewed, except in one case: One male, non-minority participant attended both workshops and was interviewed twice, once after completing each workshop. We decided to include him in the sample because, on the one hand we had a limited number of male participants, and on the other, for an opinion of one participant who could speak to both workshops and compare them. The interviews were recorded and transcribed and the data coded on the basis of a mixture of a priori categories derived from the questions asked and grounded theory methods to see what emerged from the data as we were coding and analyzing it. Our main question in the analysis was to see if mindfulness was a useful tool in equity training, whether mindfulness exercises would make participants more responsive and/or sensitive to the ideas of equity and fairness being related to compassion and in what way.

The comparison group ( 8 respondents, 3 male and 5 female, 1 male minority, 1 female minority - both racialized - and 1 male non-visible minority) completed the first workshop where participants were given theory and information about equity and compassion, definitions of equity vs. equality, videos on gender equality, the importance of compassion, a role play exercise on inequality, and reflective exercises about the social location of people in 
literature excerpts, as well as their own social location. The one role play exercise used in this workshop, "the race of life" illustrates inequality experientially by using a race-track as a metaphor for the cultural idea that we are in a life-long race seeking economic success in capitalist societies. Participants are asked to imagine that they are on a race track with a start line where individuals have no assets, and at the finish line all the assets for a successful life in modern society have been achieved (whatever this is in participants' imagination). Each volunteer that comes up to the front to enact this race of life is given a few social characteristics and allowed to keep their own gender identity (for example, "you are the child of the Prime Minister," or "the racialized child of a young homeless mother who relies on food banks and shelters for housing," or "the child of a construction worker who is a project coordinator, who therefore has more experience and skills than other construction workers," or "the disabled child of a successful lawyer" and so on). They discussed whereabouts on the start line they would be situated as children and then physically position themselves along the line. This exercise was done in both workshops. Another exercise that was completed in both workshops was their introduction to the wheel of diversity and the primary and secondary sources of our identity. In the comparison group, participants were provided with excerpts of individual life-stories of specific characters and were asked to reflect on the social location of these characters. Later, they were invited to reflect on their own social location according to the wheel of diversity. The rest of the comparison-group workshop provided a forum for discussion of information, definitions and illustrations of equality, equity, the complexity of identity, and the importance of compassion.

The target group ( 9 respondents, 2 male, 7 female, 1 male minority) engaged in a mindfulness approach equity workshop, where the same theory and information was provided to them, they saw the same videos on gender equality (excluding the videos on compassion) and completed the same role play "the race of life" on inequality and the self-reflective exercises on social location; instead of discussing compassion on the basis of the information and videos, this workshop also involved participants in mindful meditation, a series of mindfulness exercises, and an empathy-fostering imaginative exercise. These exercises stressed an awareness of our own emotions as well as the importance of compassion towards other and self-compassion. My research assistants and I designed an empathy-fostering exercise around the idea of "Sonder" as a preamble to mindfulness; the realization that each random person that goes by is living a life as vivid and complex as one's own (Koenig, 2012). In this exercise, participants were asked to see a clip from a famous movie (Titanic), then introduced to the concept of "sonder," and then invited again to see the clip focusing on an imaginative exercise about passersby in the scene, what the richness and complexity of their lives could be instead of focusing on the protagonists of the movie. A definition of mindfulness was presented to participants of this target group as: "Relating to one's experience within an orientation of curiosity, experiential openness, and acceptance. Gaining insight into our own mental, emotional, and bodily states. De-centering one-self, seeing one's own thoughts and feelings as relative and temporary. Mindfulness fosters compassion and self-compassion." After this, they were invited to complete a mindfulness inventory (Walach et al., 2006), to give them an idea of how mindful they had been for the last 48 hours, and then led through a guided mindful meditation. This prepared them to be shown pictures of diverse populations representing minority life-styles and social locations (for example, as a series of couples kissing: a white heterosexual couple, a gay couple, a heavy set couple, a

*The original version of this exercise was developed by my colleague Dr. Dawn Farough, for her social inequality course in sociology in the Faculty of Arts, TRU. 
trans couple; images of police brutality towards Caucasians and minorities, images of racialized minorities being oppressed and images of people wearing different ethnic garments) and participants were invited to notice and record their reactions and emotions elicited by these pictures. At the end of the workshop participants were asked to write compassionate letters to themselves.

\section{Findings and Discussion}

\subsection{Low Self-Awareness}

In reading the transcripts for both groups of respondents, it became clear that there was a qualitative difference between the two groups in how they reported their impressions on the topics of inequality, privilege, disadvantage, and the importance of emotions, guilt, and compassion in equity work. Participants in the comparison group in which mindfulness exercises were not used appeared at times to be unaware of their own prejudice, and when self-reflective, they reflected about their own identity without looking as closely at their own privilege. They also reported that other people needed to be more self-aware about their involvement in perpetuating privilege and disadvantage. It was interesting to see that the respondents in the comparison group that talked about feelings of guilt were respondents that belonged to minority groups who had received accommodations due to their minority status.

A measure of a person's awareness about their own involvement in perpetuating inequality could be found in self-reflection, which was expressed in both groups-but again, with differences in quality, tone and in focus of self-awareness. For example, one of the respondents in the comparison group either did not internalize or disagreed with the idea that nobody is immune to prejudice, even though this was a major topic discussed in the workshop that this participant completed. When talking about self-awareness, this participant said:

I've always had an open mind, I don't notice color, creed, nationality, so much. I look past that. I like to be with people, so really the rest of it is just a shell. You know what I mean?

Some respondents in the comparison group reported self-awareness about their own identity and social location, but also exhibited resistance to an awareness of their own privilege, illustrated by statements such as:

I think one of the ones which leaps out more and I've thought a lot more-sinceabout, is my lack of identity of where I'm from...

I know I come from the white privileged life, white privileged male-but there's a lot of us who don't do very well in life at all...

Another participant in the comparison group said it is important to

...remind ourselves of the privilege that we were actually born into, and really appreciate, like: 'wow, we are so fortunate'

However, this kind of self-awareness appeared superficial and too general when contrasted to that of the participants in the target group, discussed and illustrated below. 
As mentioned above, two of the respondents with minority status who had received accommodations expressed not feeling at ease for getting a break:

So I guess there was a bit of guilt on my side that I was receiving funding, that my other classmates were not; even though they were expressing the need for further supports.

I get a little uneasy sometimes telling people what I do because ... maybe people kind of look at it like: 'oh, how come this group of people gets this?'

People belonging to privileged groups in this comparison group of workshop participants did reflect on their unearned privilege, as mentioned above, but in terms of being fortunate, and not related to guilt; sometimes making it a matter of other people needing to be more selfaware (not one-self).

\subsection{Increased Self-Awareness}

The one category that emerged from the data, which was very revealing of the contrasting impressions that participants got from the two different workshops, was the quality of their self-awareness, about their own identity and also about their involvement in perpetuating privilege or disadvantage. In contrast to the comparison group, participants in the target group that engaged in mindfulness exercises, were more self-reflective, tended to put themselves in other people's shoes, considered their own involvement in perpetuating inequality in a personal way, expressed guilt about unearned privilege, but also stressed the importance of caring for one-self. Respondents in the target group expressed self-awareness in terms that were more involved and more empathetic. This was revealed, for example, in expressing judgement of equal possibilities (that I could find myself in the same situation):

...you have to understand that they would be-a lot of them-in that same position, just because, what if that happened to me?

I find myself looking at people and wondering what their story is; I wonder what's happening with them-people who are happy, people who are sad...

Participants in the target group also reported empathic thinking and compassion in their concern for other people; for example, one respondent in this group said:

We're talking about, not only knowing me and having compassion for my own place, but also knowing a bit about you, having the ability to ask and to enquire, as well as being compassionate then.

\subsection{Awareness of One's Own Bias}

One way in which keen self-reflective awareness was shown in the target group was through discussion of their own personal privilege or prejudice, posed in a personal way, and even revealing guilt. When being self-reflective about their own prejudice participants in the target group seemed to have handled it better and did not deny it. This statement from a target group 
respondent summarizes this point: "I just had key takeaways for myself saying-Okay, maybe I am biased in this."A set of statements from four different participants in the target group reveals similar kind of awareness:

Was it the one with the two guys kissing? ... And then there was two heavier set people kissing - and I noticed that. And then I thought, "Oh, why am I noticing that?" Love is love, right? So you do a self-check.

... to just be mindful of different things that people go through and to recognize my own bias, 'cause that was a key take away as well that, of course, we may not see it all the time but there's definitely a bias based on so many different factors.

I can sit and tell you, I would say "I'm not a prejudiced person," but realistically, I am. So that made me a little upset, but it is-that's realistic. I'm not profound at it, it's not exaggerated, but maybe it is. Again, maybe I'm just naive about it. It's something I'm still thinking about it... That's what I'm seeing, this circle of, "No I'm not prejudiced, but yes I am."

Well, when I left that workshop, I was feeling pretty... almost enlightened I would say. Because I'd say that I try to be a very equitable person, but that little exercise around the movies and having men versus women, and I thought we had come a long way as far as the gender disparity, but when that was pointed out, I took it home into our personal life...

There was a clear and conscious contrast in some participants between knowing about equity intellectually versus a keen self-awareness that comes from empathy. And participants in the target group commented on how this perspective elicited more self-doubt, as is revealed in the four statements listed above and also very clearly in this following statement:

I seem to think I'm quite aware, but am I? Maybe I'm doing something and I'm not aware of it. That's my fear.

I close this section by quoting the one respondent that participated in both workshops in the comparison and target groups. When asked to compare the first workshop (comparison group) with the second workshop (target group), he said about his own awareness:

I think this time I was probably more aware of my position of privilege...the first one was probably a bit more theoretical about knowing our identity and looking at what that means about us which, like I say, I got tied up a little-probably a lot more in my identity - and I couldn't place myself. Whereas this time, yeah I think thinking about other people [...] I'd never actually realized when in equal circles, I've still got that, just automatic level of power.

\subsection{Compassion and Self-Compassion}

In both workshops the importance of compassion and self-compassion was discussed, but this seems to have sunk in more clearly in the target group who practiced mindfulness. Here are two statements describing the effects of the mindfulness exercises in the target group's 
workshop:

It's like putting yourself in somebody else's shoes, doing by participation, that's what I'm trying to say, you feel part of it, and then you're thinking, and then it enhances your thinking differently.

It goes back to knowing something intellectually versus actually putting yourself in there. I'm talking about it from a more objective point of view [laughter] so it was good. It was a really valuable exercise.

Referring to their perspective as more "objective" in the sense of more "real." There were also reflections on how important it is to care for one self in a culture where there is a high esteem placed on hard work and personal sacrifice:

'Cause you're always, "Well I don't want to do this but it's for you so yes, I'll do it." But then, your bucket gets empty and empty and empty, so then you end up completely stressed-out, running crazy, right?

...we're always hardest on ourselves, right? For sure, I mean I often wish I could do more, or be more, and when I'm not, I'm always hardest on myself. But then when you're in that mindset, then it is hard to be compassionate and look outwards to others because you're in that spot.

There was awareness that it is important for us to look after ourselves, to have selfcompassion. One participant in the target group said:

So I say if you care about the family, and you put everyone in a circle, then you are also taking part in that circle, right? And if you don't care about that part of the circle, then you are doing damage to the circle. You've got to look after yourself, and without looking after yourself, you cannot look after others.

\section{Conclusions}

In thinking about the importance of a complex identity and also of compassion to equity work, I looked for effective tools for the purposes of equity training (anti-oppression, anti-sexism, anti-racism training). The findings of this pilot study have shown that resistance to this kind of training can be reduced by increasing self-awareness and awareness of one's own bias on the basis of mindfulness and self-compassion. This was accomplished by using mindfulness exercises and also exercises designed to improve an empathic identification with the position of the "other" (such as the "Sonder" exercise explained above), and in discussing these concepts beyond their abstract meaning. Mindfulness and mindful meditation became key tools in designing exercises that would promote empathy, contact participants' own emotional involvement with prejudice, as well as helping them be more self-reflective and self-aware. What I observed when I integrated mindful meditation and exercises that promoted this kind of mindfulness was that participants responded in the debriefing periods with a keener awareness of their own involvement in perpetuating inequity (self-awareness), expressed concern about their role in creating undeserved structures of privilege, displayed clearer empathy towards people who were located in disadvantaged situations, and expressed that 
both caring for others and for one-self were important in achieving equitable goals.

This is the reason why I decided to explore these possibilities in research and thus how this pilot project came to fruition to more systematically test these impressions. This project has allowed me to preliminarily test how useful mindfulness exercises can be in engaging participants with equity work as self-awareness and as a "labor of love." Nevertheless, this pilot study is not conclusive; more research needs to be done at a larger scale, to explore this approach to equity training further. I plan to design and engage in a larger study that will involve interviews as well as focus groups with participants. I will also explore the possibilities of online distance education for equity training in the form of an online workshop.

Today it has become urgent to engage in equity and anti-oppression work with love and not anger. The latter had its place in the history of discovering the importance of resistance and struggle against oppression. But activism ought to leave anger behind. Anger leads to violence, which leads to further fear and oppression. A collective life with others in society that is free from hate and fear is what I have referred to as a labor of love: We all have an important role in building our lives together in our communities, our places of work, our public spaces. In this labor of love, it is of the essence to apply mindfulness about our own involvement in perpetuating stressed communities due to prejudice and discrimination, engage with our guilt and discomfort due to our own privilege, and then drop the guilt through compassion and self-compassion. This kind of work helps us see that all human beings are fallible, we share in this common humanity, and we owe each other this effort in building diverse communities that live in freedom from hate and fear.

\section{Acknowledgements}

I would like to acknowledge the devoted engagement of my research assistants Nicola Tabata and Joshua Woodcock, without whose dedication to this project I could not have completed it. I am also grateful to my research students Radha Ortiz and Matthew Arkinstall for their proofreading of this article. Assetou Coulibaly was also very helpful in starting the production of the online component of this equity training project, which is still under development. This research project was completed with the funding support of the Thompson Rivers University Open Learning Research Award.

\section{References}

Anthias, F. (2012). Intersectional what? Social divisions, intersectionality and levels of analysis. Ethnicities, 13(1), 3-19.

Brookfield, S. (2014). Teaching our own racism: Incorporating personal narratives of whiteness into anti-racist practice. Adult Learning, 25(3), 89-95.

Carni, S., Petrocchi, N., Del Miglio, C., Mancini, F., and Couyoumdjian, A. (2013). Intrapsychic and interpersonal guilt: A critical review of the recent literature. Cognitive Processing, 14(4),333-346.

Choudhury, S. (2015). Deep diversity: Overcoming us vs. them. Toronto: Between the Lines.

Collins, P. H. (2000). Black feminist thought: Knowledge, consciousness and the politics of empowerment. (2nd ed.) New York: Routledge.

Dhamoon, R. K. (2011). Considerations on mainstreaming intersectionality. Political Research Quarterly, 64(1), 230-243.

DiAngelo, R. (2011). White fragility. International Journal of Critical Pedagogy, 3(3), 57-70.

Flook, L., Goldberg, S. B., Pinger,L., and Davidson,R. J. (2015). Promoting prosocial behavior and selfregulatory skills in preschool children through a mindfulness-based kindness curriculum. Developmental Psychology, 51(1), 44-51.

Galante, J., Galante, I., Bekkers, M., and Gallacher, J. (2014). Effect of kindness-based meditation on health and well-being: A systematic review and meta-analysis. Journal of Consulting and Clinical Psychology, 82(6), 1101-1114.

Gnanadass, E. (2014). Learning to teach about race: The racialized experience of a South Asian American 
feminist educator. Adult Learning, 25(3), 96-102.

Goetz, J. L., Keltner, D., and Simon-Thomas, E. (2010). Compassion: An evolutionary analysis and empirical review. Psychological Bulletin, 136(3), 351-374.

Gopaldas, A. (2013). Intersectionality 101.Journal of Public Policy, 32(special issue), 90-94.

Hernandez-Wolfe, P., and McDowell, T. (2012). Speaking of privilege: Family therapy educators' journeys toward awareness and compassionate action. Family Process, 51, 163-178.

Holas, P. and Jankowski, T. (2013). A cognitive perspective on mindfulness. International Journal of Psychology,48(3), 232-243.

Horn, J. D.(2008). My reflections on the path to uncovering my whiteness and unlearning racism. Transformative Dialogues: Teaching \& Learning Journal, 2(2), 1-5.

Jones, S. R.(2009). Constructing identities at the intersections: An autoethnographic exploration of multiple dimensions of identity. Journal of College Student Development, 50(3), 287-304.

Koenig, J. (2012). Sonder. The dictionary of obscure sorrows. Retrieved from http://www.dictionaryofobscuresorrows.com/post/23536922667/sonder

McIntosh, P.([1988] 1990). White privilege: Unpacking the invisible knapsack. Independent School, 49(2), 3135.

Navarrete, F. (2016). México racista: Una denuncia. Mexico City: Grijalbo.

Neff, K. D. (2003). Self-compassion: An alternative conceptualization of a healthy attitude toward oneself. Psychology Press, 2, 85-101.

Neff, K. D., Kirkpatrick K. L., Rude S. S. (2007a). Self-compassion and adaptive psychological functioning. Journal of Research in Personality, 41,139-154.

Neff, K. D., Rude S. S., and Kirkpatrick K. L. (2007b). An examination of self-compassion in relation to positive psychological functioning and personality traits. Journal of Research in Personality, 41, 908-916.

Neff, K. D. and Germer C. K. (2013). A pilot study and randomized controlled trial of the mindful selfcompassion program. Journal of Clinical Psychology, 69(1), 28-44.

Nussbaum, M. (2001). Upheavals of thought: The intelligence of emotions. Cambridge, U.K.: Cambridge University Press.

Sánchez-Flores, M. J. (2005). Political philosophy for the global age. New York: Palgrave Macmillan.

Sánchez-Flores, M. J. (2010). Cosmopolitan liberalism: Expanding the boundaries of the individual. New York: Palgrave Macmillan.

Schonert-Reichl, K. A., Oberle, E., Lawlor, M. S., Abbott, D., Thomson, K., Oberlander, T. F., and Diamond, A. (2015). Enhancing cognitive and social-emotional development through a simple-to-administer mindfulness-based school program for elementary school children: A randomized controlled trial. Developmental Psychology, 51(1), 52-66.

Spectrumlens. (2008, April 26). Unpacking the invisible knapsack [video file]. Retrieved from: https://www.youtube.com/watch?v=DRnoddGTMTY

Walach, H., Buchheld, N., Buttenmuller, V., Kleinknecht, N., and Schmidt, S. (2006). Measuring mindfulnessthe Freiburg Mindfulness Inventory (FMI). Personality and Individual Differences, 40(8), 1543-1555. 